SSC20-II-03

\title{
On-Orbit Performance of the BCP-100 Green Propellant Infusion Mission
}

\author{
Brian Marotta, Christopher McLean, Brad Porter \\ Ball Aerospace \& Technologies Corp. \\ 1600 Commerce St, Boulder, CO 80301; 303-939-4972, 303-939-7133, 303-939-4103 \\ bmarotta@ball.com,cmclean@ball.com,bporter@ball.com
}

\begin{abstract}
The Green Propellant Infusion Mission (GPIM) spacecraft was launched in June of 2019 as a secondary payload on the Air Force's STP-2 Falcon Heavy launch vehicle. GPIM is a BCP-100, a line of ESPA class spacecraft designed by Ball. The BCP-100 was designed around the capability to support multiple payloads on a single platform, and be able to fly in a wide range of orbits without the need to reconfigure the spacecraft in any way. Proving the flexibility and multi-role capability of the BCP-100 design, there are three secondary payloads on GPIM. In addition to the green propellant payload, iMESA, SWATS, and SOS are hosted on the spacecraft. These were provided by the Air Force through their Space Experiments Review Board (SERB) payload list. Finally, an advanced multi-layer insulation (MLI) technology, integrated MLI (IMLI), was employed as insulation for the propulsion subsystem. This technology was developed by Ball and Quest Thermal Group under Small Business Innovative Research (SBIR) funding provided by NASA STMD. GPIM adds to the 15 years of combined flight time for the BCP-100 line.
\end{abstract}

Ball Aerospace built the GPIM spacecraft for NASA's Space Technology Mission Directorate (STMD) in order to provide a platform to accomplish on-orbit testing and validation of an AF-M315E based green propellant propulsion subsystem. After a very successful spacecraft commissioning phase that was completed in less than 24 hours, check out of the primary green propellant payload commenced. This included testing the primary and redundant catbed heaters, opening the latch valve, and commanding the thrusters in both open loop and closed loop control modes, all of which was accomplished within three and a half days of being on-orbit.

The propulsion system employs five protoflight $1 \mathrm{~N}$ thrusters, four for attitude control and the fifth for use during delta-v burns to provide higher thrust. Characterization of the green propellant system has been on-going. This characterization includes performing closed loop delta-v burns, 3-axis thruster-based attitude control, and momentum dumping. In addition to these tests, on-orbit measurement of the thruster impulse-bit has been performed over the course of the mission. This measurement involves a complex command sequence in which the spacecraft must execute a delta-v, perform multiple maneuvers, spin down the reaction wheels, and execute a series of $200 \mathrm{msec}$ long open loop thruster pulses. Analysis of the spacecraft motion that results from each thruster pulse provides the amount of force that was applied.

This paper provides a brief background of the GPIM program, including objectives of the technology demonstration, and presents on-orbit flight results of propulsion tests performed to date.

\section{INTRODUCTION}

The Green Propellant Infusion Mission (GPIM) (Figure 1) was first proposed by Ball Aerospace in 2012 as a mission to further the development of a green propellant propulsion system and provide NASA an on-orbit test bed to demonstrate the capabilities of this new propulsion technology. With a program award in late 2012, Ball proceeded to build upon the flexible Ball Configurable Platform (BCP)-100 spacecraft bus design to include the capability of performing a wide range of propulsive operations, including delta-v, attitude control on thrusters, thruster based momentum dumping, and open loop thruster control. GPIM has been managed by the NASA TDM program office at the Marshall Space Flight Center, which is responsible for demonstration projects that bridge the gap between technology development, and the infusion of system-level technologies into missions undertaken by industry, NASA, and the DoD. The green propellant technology promises higher performance for future satellites by providing options for longer mission durations, additional maneuverability, increased payload, and simplified launch processing.

By leveraging experience gained in building STPSat-3, and minimizing the number of component changes, the GPIM bus had a short integration and test (I\&T) phase, taking roughly 50 days to go from electrical integration through bus complete. The I\&T campaign completed in late 2015, when the spacecraft was put into storage. GPIM was one of multiple EELV Secondary Payload 
Adaptor (ESPA) payloads slated to get launched on STP2, a Falcon Heavy qualification flight for the Air Force. Due to development delays in having the Falcon Heavy ready for launch, GPIM was in storage for approximately 3.5 years.

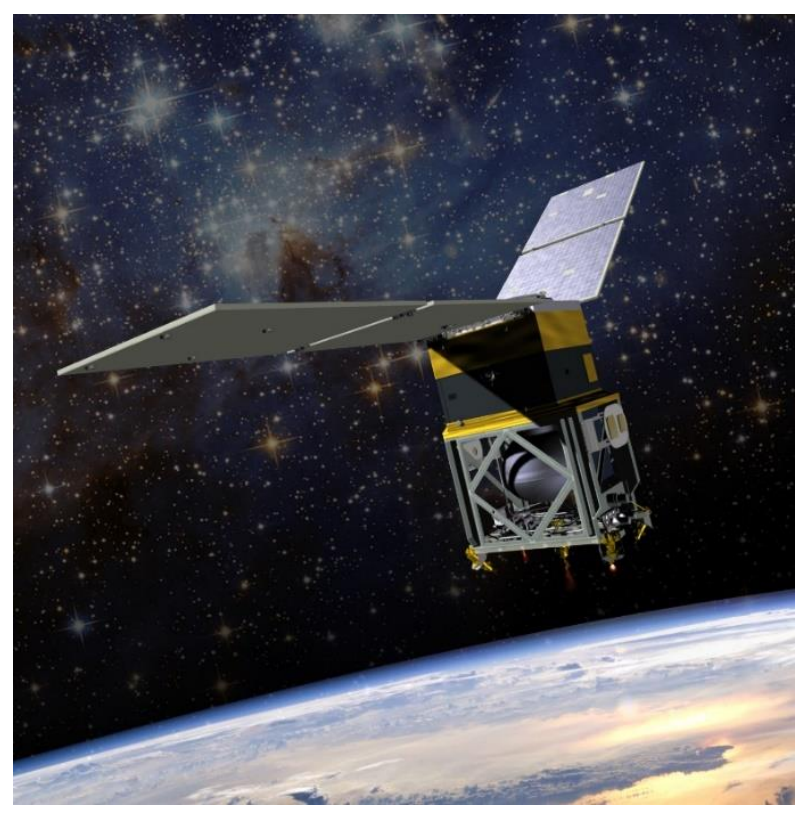

Figure 1: GPIM Flight System

Launch occurred on June 25, 2019, and with the successful deployment of all payloads on the STP-2 manifest, GPIM was on-orbit, getting deployed at 07:57:14 UTC at an altitude of $721 \mathrm{~km}$ and an inclination of 24 degrees. The Ball mission operations team was comprised of engineers that had also supported the STPSat-2 and STPSat-3 launches, and applying that knowledge and experience to GPIM's early orbit campaign resulted in a very quick and efficient checkout of the spacecraft. Three days after launch, the first step of the thruster checkout was performed, with a series of open loop commands executed to fire each of the 5 thrusters for a fixed amount of time. This was followed less than 24 hours later by the first closed loop test, a short delta-v that applied 0.13 meters-per-second of change to slightly lower the perigee altitude. In the ensuing months, additional thruster operations have further lowered the perigee altitude, tested the pointing accuracy while on thruster attitude control, performed multiple thruster characterization tests, and shown the ability to manage reaction wheel and spacecraft momentum.

\section{SPACECRAFT DESIGN}

\section{Space Vehicle and Propulsion Subsystem}

GPIM is built around the flexible Ball Configurable Platform (BCP)-100 spacecraft bus. Ball Aerospace's
BCP-100 spacecraft bus features standard payload interfaces, robust power and thermal design for any LEO orbit, and a heritage Command and Data Handling system. The BCP-100 is an assembly of five component panels, with the sixth side of the bus being the Payload Interface Platform (PIP). The PIP was used as the structural platform for the assembly of the propulsion subsystem, and was provided by Ball Aerospace to Aerojet Rocketdyne, the mission partner responsible for manufacturing the propulsion system.

In Figure 2, the GPIM space vehicle is shown with the BCP-100 bus and the GPIM propulsion payload. On the upper deck of the propulsion subsystem, the five $1 \mathrm{~N}$ thrusters are mounted, indicated as rocket engine assemblies (REA) 1-5 (Figure 3). REA's 1-4 are the attitude control system (ACS) thrusters and REA 5 is the delta-v thruster. The attitude control thrusters are steeply canted to maximize the moment-arm between the ACS thrust vectors and the center of gravity of the spacecraft, facilitating resolution of the impulse measurement. REA 5 is only used during orbit adjustment operations. Developed by the Air Force Research Laboratory (AFRL) at Edwards Air Force Base, CA, the green propellant for GPIM is AF-M315E, a blend of hydroxylammonium nitrate (HAN) and other ingredients, and it offers nearly 50 percent higher performance for a given propellant tank volume compared to a standard hydrazine system. In addition to higher performance, AF-M315E is a low toxicity chemical, which provides much higher factors of safety for fueling operations compared to hydrazine.

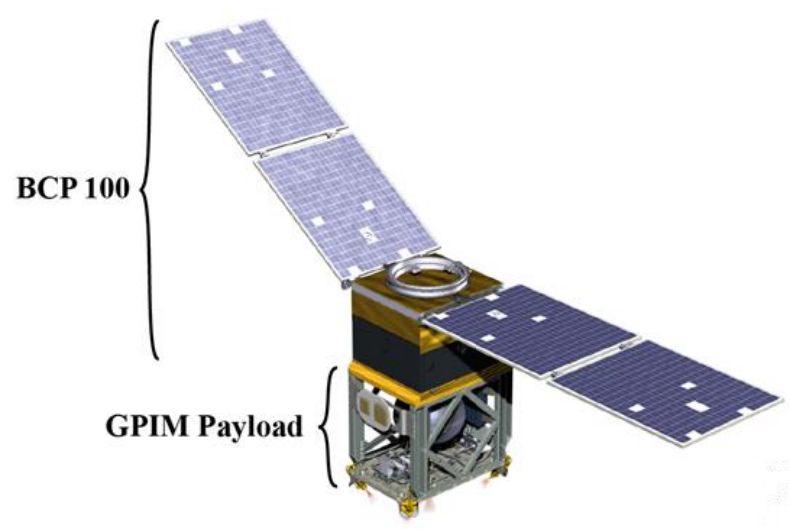

Figure 2: The GPIM Flight System 


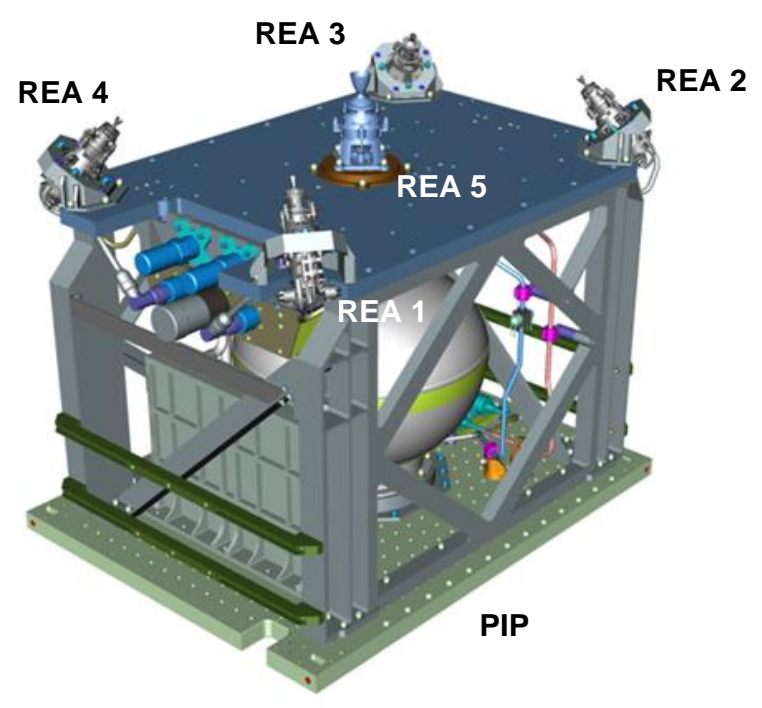

Figure 3: Propulsion Subsystem Showing Thruster Definitions

\section{GPIM Space Vehicle Flight Coordinate System}

The ADCS coordinate system and rotational axis are shown in Figure 4. Nominal flight direction is with the $+X$ axis in the direction of the orbit velocity vector, and the $+\mathrm{Z}$ axis facing nadir. Orbit lowering operations occur with the $+\mathrm{Z}$ axis in the direction of the orbit velocity direction.

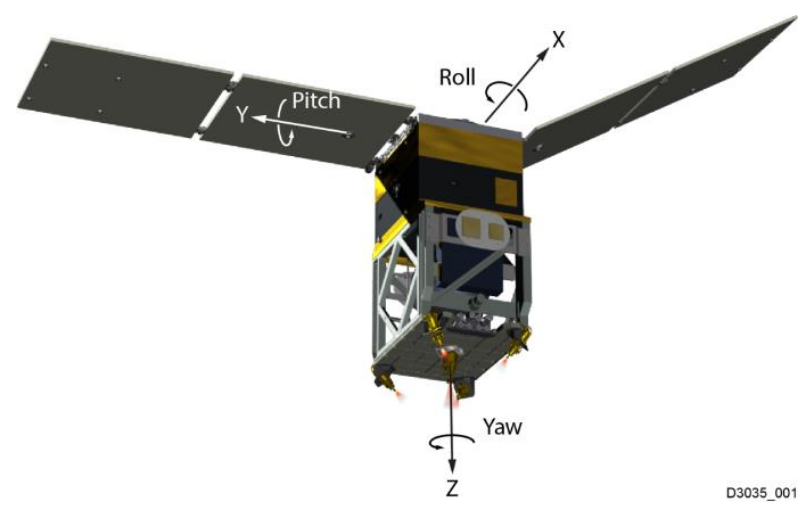

Figure 4: GPIM space vehicle with ADCS coordinate system

\section{GPIM Thruster Based ADCS Operational Modes}

Making use of Ball heritage algorithms that have been used to perform thruster operations on deep space and LEO missions, three distinct ADCS modes were added to the baseline flight software package inherited from STPSat-3. These modes offer flexible, table parameter driven thruster operations that allow GPIM to achieve the mission objectives.
Delta- $\mathrm{V}$ Mode is a closed loop mode where the spacecraft is operated with the primary divert thruster commanded to $100 \%$ duty cycle, and the four corner ACS thrusters are pulse modulated to provide thruster vector control. This mode has been employed during spacecraft checkout and used to perform the perigee lowering operations. Nominal thrust vector control occurs with the ACS thrusters operating at $80-100 \%$ duty cycle. Additionally, through a simple table parameter update, Delta-V Mode offers the ability to perform an off-modulated delta- $v$, where the ACS thrusters operate at a low duty cycle of approximately $5 \%$, while REA 5 operates at $100 \%$.

A second mode added to GPIM is Point Mode with the ability to select different actuator configurations. When configured to perform a thruster-based operation, REA 5 is not operated and the four corner ACS thrusters are used in different operational states depending upon the demonstration. For GPIM experiments, the thrusters are operated in closed loop control during the Pointing Control Demonstration and the Momentum Management Demonstration, where the ACS thrusters either control spacecraft pointing or control the momentum state of the reaction wheels. The Ibit characterization testing is performed with the thrusters in an open loop control state, executing a series of discrete pulses.

The third ADCS mode is Detumble Mode, where the ACS thrusters are fired in closed loop control to dump total spacecraft momentum. Traditionally, this mode is designed to be used in a spacecraft emergency scenario, where the reaction wheels are saturated and the spacecraft is tumbling.

\section{MISSION OBJECTIVES}

The GPIM program has an overall primary mission objective of flight qualifying a green propellant based propulsion system through a series of on-orbit demonstrations and tests. On-orbit tests have been developed to prove the capability of the green propellant propulsion system (GPPS) as well as characterize the thruster efficiency over the life of the mission across a range of tank pressures. These tests also encompass a wide range of possible thruster-based operations to exercise the system in a variety of operational modes.

Since this is a new propulsion technology, an incremental approach has been developed to first check out the system to verify initial health and configurations are as expected, before moving on to a fully operational status. Checkout consists of first performing short duration, open loop pulses on each of the thrusters in order to verify functionality and as a positive confirmation that the hardware phasing is correct. Which is to say that when REA 1 is commanded by flight 
software, REA 1 generates force. During this open loop checkout, the reaction wheels remain active in controlling the spacecraft attitude so that the response due to a thruster pulse is obvious, as the reaction wheels will correct any attitude error the thruster pulses create.

Following the open loop checkout, the first closed loop checkout will be performed, in which the spacecraft will be commanded into Delta-V Mode. All five thrusters will be operated at desired duty cycles for a short duration of 5 seconds. The reaction wheels are held at a constant speed, and thrusters are used to control spacecraft attitude and rates. This will help to verify the functionality of this new mode before relying on it to perform longer duration burns that are critical to the mission success. Performing this short delta- $v$ will complete the GPPS checkout phase of the mission and allow for moving into the operational phase.

One of the primary mission objectives is to measure overall thruster health and performance metrics across a range of tank pressures. This is accomplished by performing an Ibit test, which is a measure of the amount of force a thruster generates for a given pulse width. Performing an Ibit test is the first planned operational use of the propulsion system, as the tank pressure is still at the beginning of life value of 410 PSI, and will allow for mapping back to testing performed by Aerojet Rocketdyne during development of the system. In order to measure the Ibit, a series of 120 open-loop thruster pulses will be commanded, each pulse being $200 \mathrm{msec}$ long, with the control system in a fully disabled state where the torque rods and reaction wheels are not generating torques. By performing a thruster pulse for a known amount of time, and collecting star tracker attitude data during the drift period, analysis can be performed to back out the amount of thrust applied that resulted in the measured motion of the spacecraft. Also key to performing this test is using the ACS thrusters to spin down the reaction wheels and letting the spacecraft start from as quiescent state as possible. Measuring the Ibit on-orbit throughout the mission will allow for thruster performance characterization as the propulsion system ages, so this test will be repeated after each large change in tank pressure, i.e., after each large change to the orbital altitude is performed.

The next most important objective in proving this new technology is performing longer duration delta-v's. In continuing to follow a measured approach to operations, a 4 minute long delta-v will continue to build confidence in the system performance and also allow for an opportunity to determine whether any flight software table parameters need to be tuned to optimize performance. Once this delta- $\mathrm{v}$ is completed and the performance is analyzed, the first of four planned orbit lowering campaigns will be performed. Each campaign is comprised of multiple longer duration burns, lasting anywhere from 6 to 9 minutes, depending upon how many burns are planned for each campaign. Following the optimized flight plan shown in Error! Reference source not found., four different perigee altitudes are targeted. This allows for the Ibit measurement to be performed at different tank pressures as the system ages.

Another set of objectives is performing a series of attitude control demonstrations. The first of these demonstrations is to control the spacecraft attitude and rate on thrusters. This will be accomplished by commanding the actuator configuration to disable the reaction wheels and torque rods and enabling the thrusters to control the attitude and rate. The next planned demonstration is to allow the ACS thrusters to control system momentum by disabling the torque rods and sending momentum error commands to the thrusters. While this is a capability that would not be required for an Earth orbiting spacecraft, it is a function that a GEO or interplanetary spacecraft would need to perform, and it will further demonstrate the versatility of this propulsion system. The final attitude control demonstration planned is to induce some amount of momentum into the system and then command ADCS into the Thruster Detumble Mode, which will quickly dump that momentum. This would demonstrate the ability to remove a level of system momentum that has saturated a spacecraft's reaction wheels and caused the spacecraft to tumble, with the objective of returning the system to a level in which the wheels could be used again. After a peer review of the operational plan was done by the propulsion group at Goddard Space Flight Center, one additional demonstration was added in which a short delta-v would be performed with REA 5 commanded at $100 \%$ duty cycle, and the four ACS thrusters set to an off-pulsing control mode.

The final objective of proving this new propulsion technology is to perform a series of burns at the end of the mission with the goal of lowering perigee to $180 \mathrm{~km}$ or less. While the system is not sized large enough to accomplish an active re-entry, achieving re-entry due to atmospheric drag within two weeks of reaching $180 \mathrm{~km}$ will be possible. Additionally, this will allow for maximizing the throughput of the propellant and the thrusters, as burns will continue until the propellant is showing signs of being fully depleted.

In addition to the primary GPPS payload, there are three experimental payloads integrated on GPIM that were provided by the Department of Defense (DoD) Space Experiment Review Board (SERB). These payloads are the Integrated Miniaturized ElectroStatic Analyzer Reflight (iMESA-R), Small Wind and Temperature 
Spectrometer (SWATS) and the Space Object Selftracker (SOS). iMESA-R is the second flight of the United States Air Force Academy's (USAFA) iMESA payload, which was flown on the STPSat-3 mission. iMESA-R is a sensor that will provide plasma density, plasma temperature, and host spacecraft charging measurements with respect to background plasma. SWATS is a Naval Research Laboratory (NRL) payload that will make in-situ, co-located measurements of the atmospheric neutral, ion density composition and winds on a global scale, and an earlier version of SWATS was also flown on STPSat-3. SOS is an Air Force Institute of Technology (AFIT)/AFRL pathfinder experiment for creating a system to decrease the chance of space collisions by having each satellite or rocket body host a similar modem that can report its position and velocity using GPS inputs on a recurring basis using a low earth orbit SATCOM constellation for up to 25 years.

Since SWATS and iMESA-R obtain higher value data at lower orbital altitudes, it was decided that instead of circularizing the orbit at various altitudes, only perigee would be lowered (Figure 5). This flight plan achieves multiple goals. First, it offers SWATS and iMESA-R the opportunity to eventually perform measurements in a range of altitudes, from $720 \mathrm{~km}$ to $300 \mathrm{~km}$, on an orbital basis. Second, as stated earlier, it will allow the ability to perform the de-orbit campaign at the end of the mission, with the goal of lowering perigee to an altitude of $180 \mathrm{~km}$.

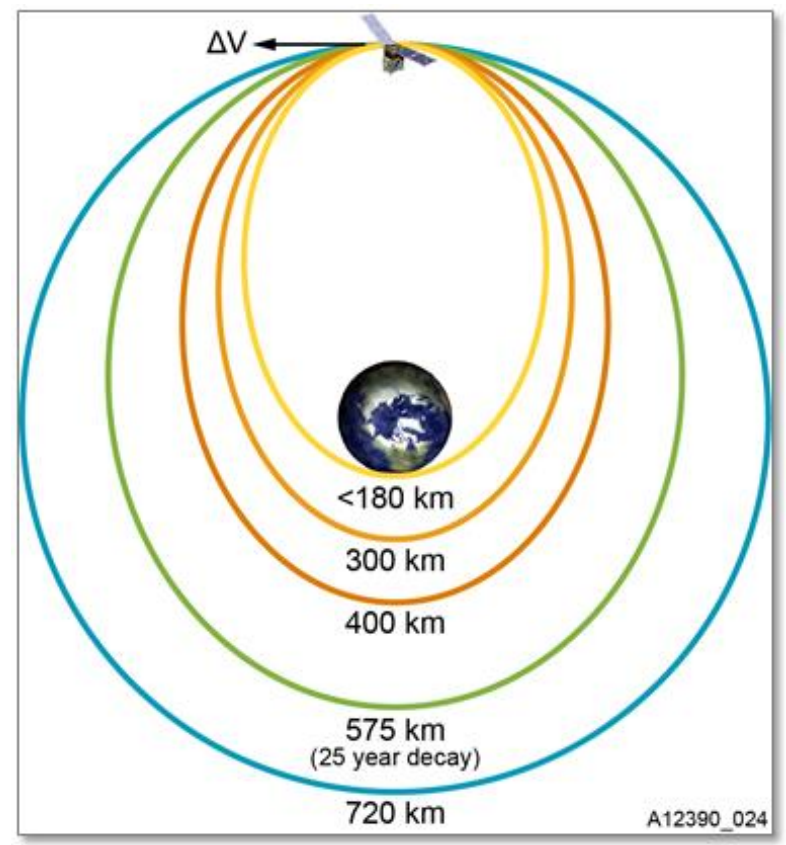

Figure 5: GPIM Optimized Flight Plan

In addition to the core green propellant technology demonstration and the three SERB secondaries, an advanced multi-layer insulation (MLI) technology, integrated MLI (IMLI), is installed on the GPPS, raising the maturity of this product to technology readiness level (TRL) 7+. This high-performance insulation technology was developed by Ball and Quest Thermal Group under Small Business Innovative Research (SBIR) funding provided by NASA STMD.

\section{FLIGHT OPERATIONS}

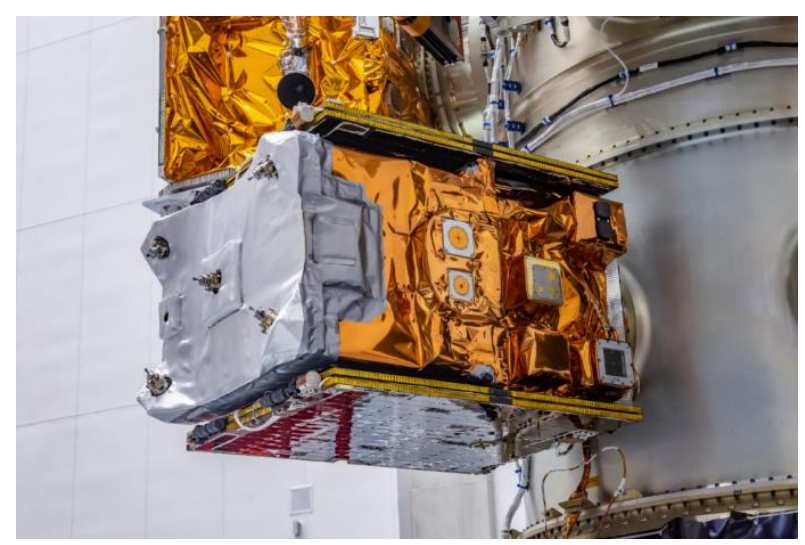

Figure 6: GPIM Integrated on STP-2 ESPA Ring NASA Photo

\section{Launch and Early On-Orbit Operations}

On June 25, 2019, at 07:57 UTC, a little under 1.5 hours after the successful launch of the STP-2 Falcon Heavy, GPIM was deployed from the ESPA ring. Roughly an orbit later, at 09:36 UTC, the first ground contact with the spacecraft confirmed that it was maintaining a stable Safe Mode attitude, with proper pointing of the solar arrays on the Sun (Figure 7).

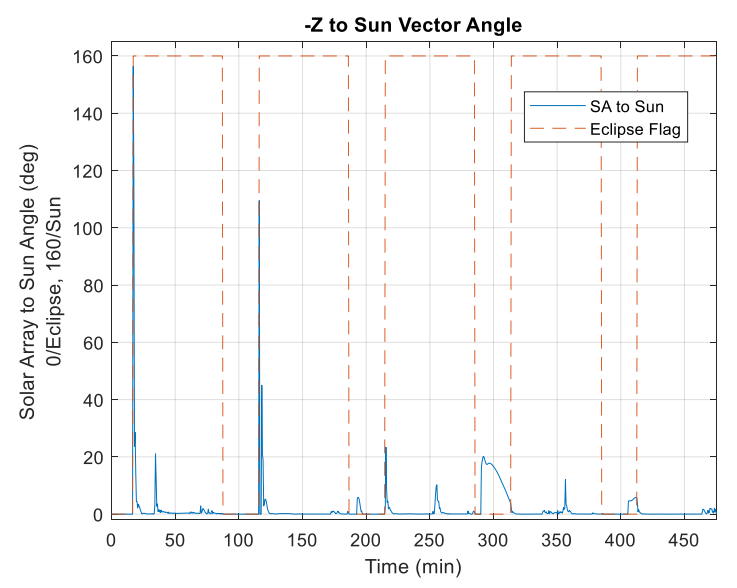

Figure 7: Solar Array to Sun Pointing for First 4.5
Orbits

With the two ensuing ground contacts, the spacecraft continued to show nominal operation of Safe Mode, and was configured for transition to 3-axis stabilized 
pointing modes. The spacecraft transitioned out of Safe Mode and into the proper orbit frame pointing mode, followed by a commanded transition into a Standby attitude to prepare for thruster catbed heater checkout (Figure 8). After some additional configurations were commanded on following contacts, bus checkout was completed less than 23 hours after GPIM was deployed from the Falcon Heavy.

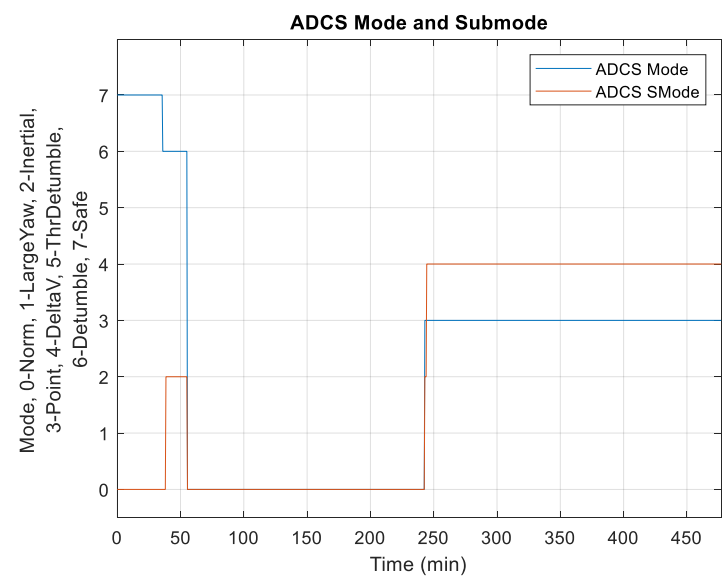

\section{Figure 8: ADCS Mode Transition from Safe (7) to Orbit (0) to Standby (3)}

The first step of the propulsion system checkout was comprised of turning on the primary and redundant catbed heaters for 60 minutes each, making the full catbed heater checkout 10 hours long. In preparation for performing this checkout, the spacecraft was commanded into a Standby pointing attitude, which offers a higher power state, as the solar arrays are yawsteered to point at the Sun. Once the catbed heater checkout was completed and the resulting temperature data was compared back to ground test data, the next step in the propulsion system checkout was to perform venting of the thruster lines. And the final step in configuring the system for operation was opening of the latch valve, which was observable in the spacecraft attitude data (Figure 9). With the successful checkout of the propulsion hardware complete, the system was configured for the first active propulsive activity, coming less than 3 days after launch.

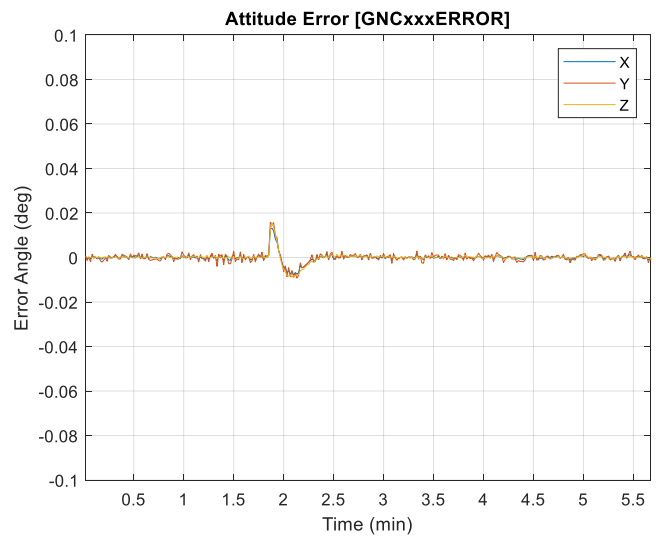

\section{Figure 9: Small Attitude Disturbance When Latch Valve Opened}

A measured, incremental approach to firing the thrusters was developed, which called for performing a series of $200 \mathrm{msec}$ long pulses (Figure 10), spaced 10 seconds apart, on each of the 5 thrusters with the reaction wheels still maintaining attitude control of the spacecraft. The open loop checkout was timed to be performed during a ground contact in order to provide the operations team real time telemetry of the checkout as it was executed. Observing the attitude error (Figure 11) and reaction wheel speeds during the test (Figure 12), it was clear that the thrusters operated and had generated some amount of torque in which the control system responded to.

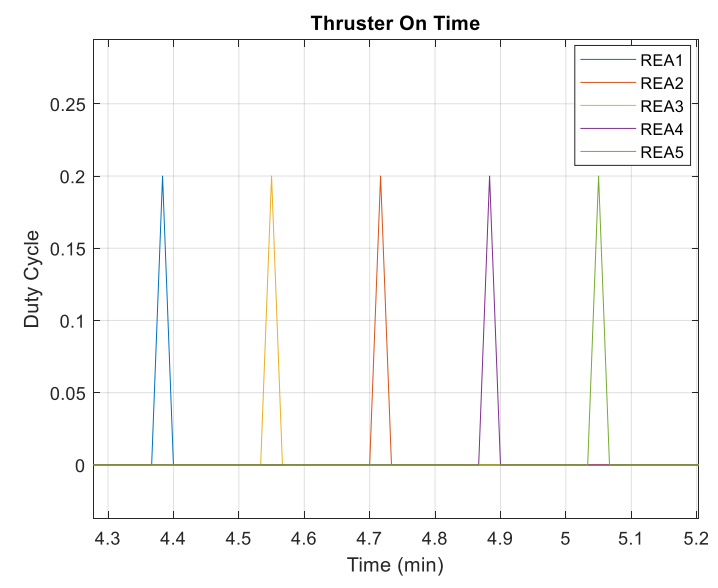

Figure 10: Open Loop Commanded Thruster Pulses of $200 \mathrm{msec}$ 


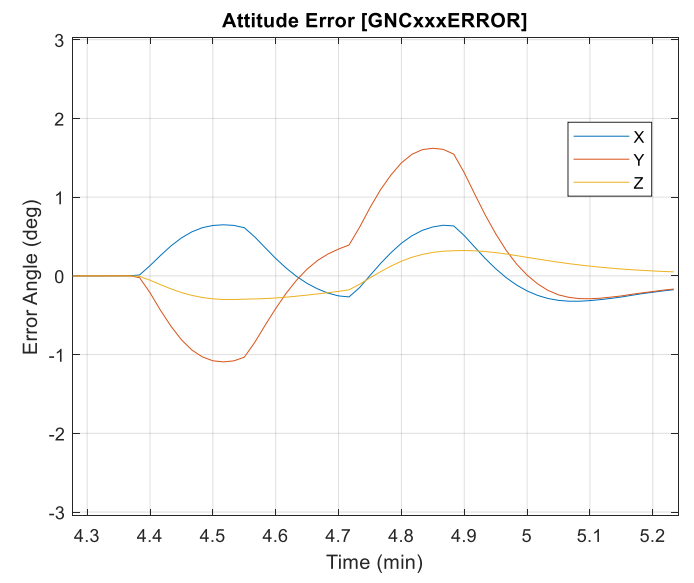

Figure 11: Attitude Error During Open Loop Thruster Firings

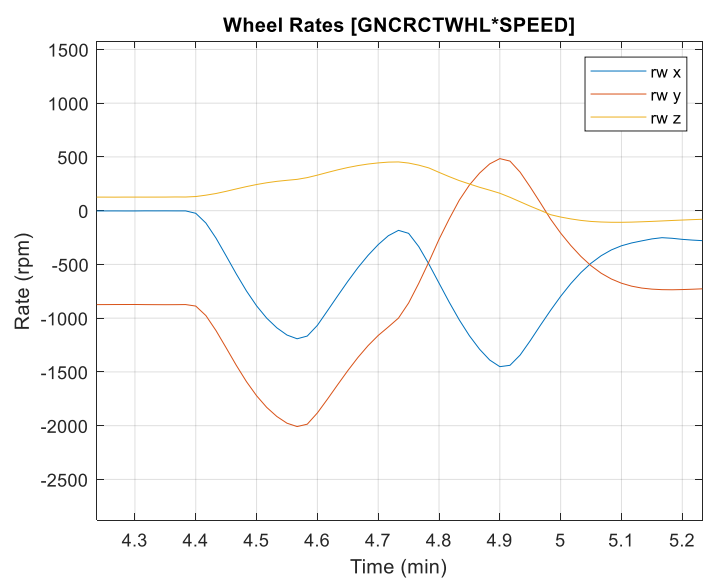

\section{Figure 12: Reaction Wheel Speeds During Open Loop Thruster Firings}

With the open loop firing of the thrusters completed, the final step required in order to declare successful checkout of the propulsion system was to perform a short, closed loop delta-v of 5 seconds. This would exercise one of the new ADCS modes added for GPIM, Delta- $\mathrm{V}$ Mode, and operate the thrusters at the higher duty cycles planned for performing the future perigee lowering burns. During this first use of Delta-V Mode, all of the thrusters were brought up to the expected duty cycles (Figure 13) and the attitude error was held to desired accuracy (Figure 14). Post processing of the orbit data by the ground support team showed that 0.127 meters-per-second of delta- $v$ had been applied to the orbit, lowering perigee by 500 meters. This activity completed the initial checkout of the propulsion system, along with allowing confirmation that the spacecraft bus was capable of performing all of the required activities for executing the mission objectives.

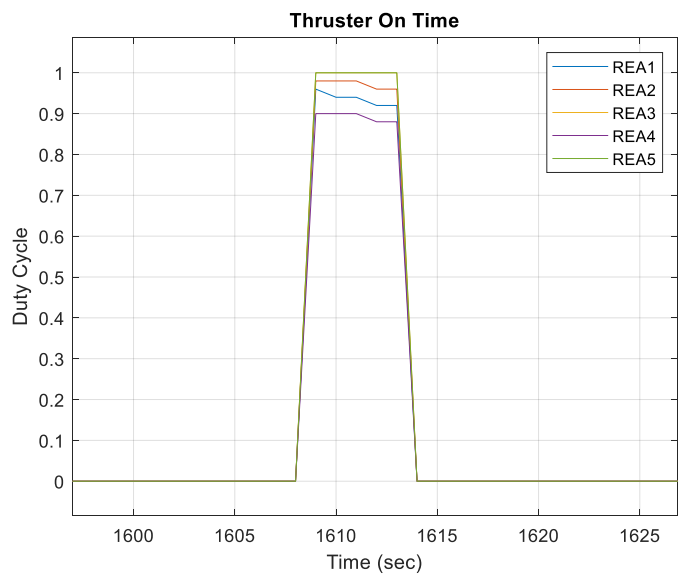

Figure 13: Thruster Duty Cycles During Closed Loop Checkout

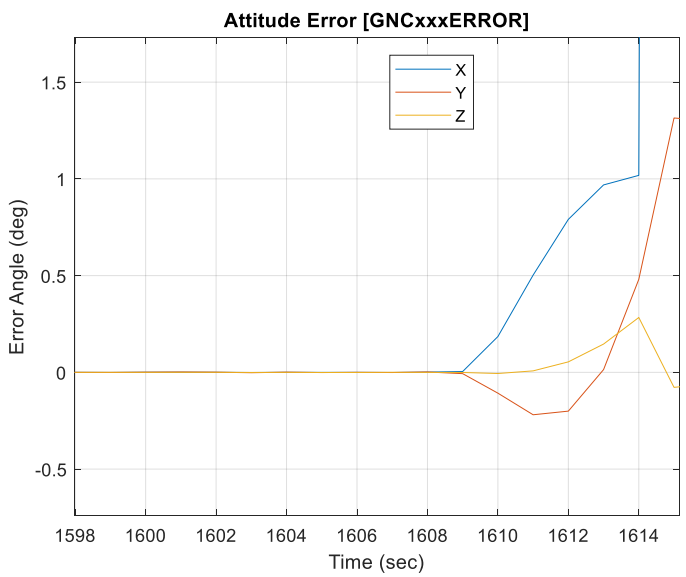

Figure 14: Attitude Error During Closed Loop Checkout

\section{Accomplishing Mission Objectives}

Prior to performing the first full Ibit measurement onorbit, the decision was made to perform an "abbreviated" Ibit measurement (Figure 15). The full Ibit measurement entails a series of complex command sequences, where a 60 second long delta-v is performed on REAs 1-4 to heat them up to operational temperatures, followed by spinning the reaction wheels down to 0 RPM, and then going directly into the 120 open loop thruster pulses. All of this is done in eclipse so that as the spacecraft rotates due to the open loop pulses, only the Earth needs to be avoided in order to maintain a valid star tracker measurement for the duration of the test. 


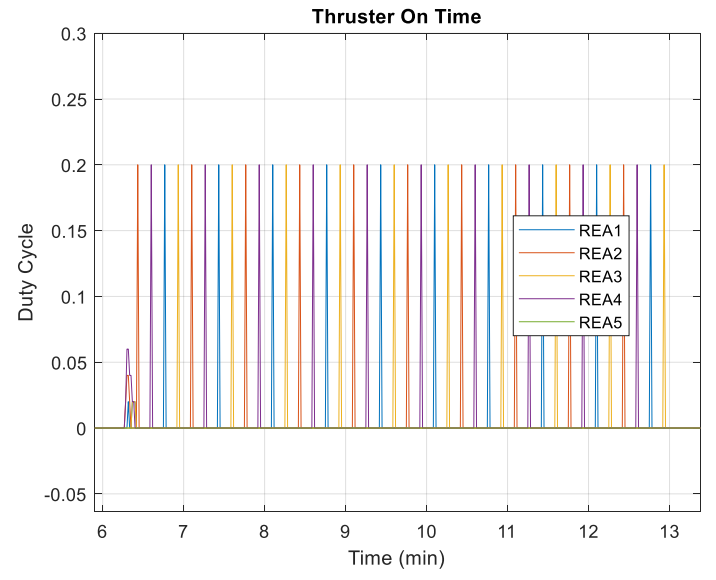

Figure 15: Commanded Thruster Duty Cycles for Abbreviated Ibit

In order to provide confidence that the proper thruster durations and starting attitude were used, the abbreviated test was designed to execute 40 open loop pulses of 200 msec each. Results of this test would then allow for tuning of the thruster pulse durations prior to executing the full Ibit measurement. The successful completion of the abbreviated Ibit test proved that the commanding sequence developed to properly configure the system for a full Ibit measurement would work. In quick succession, a 60 second long delta-v was performed on just the 4 corner thrusters to warm up them up to operational temperatures, followed by a spin down of the reaction wheels to 0 RPM (Figure 17) with the thrusters maintaining attitude control of the spacecraft. This then allowed for a low starting body rate to begin the Ibit measurement sequence, where each thruster pulse imparted a torque, and the resulting body rates (Figure 16) from each thruster firing could provide the data needed to back out the observed thruster torques.

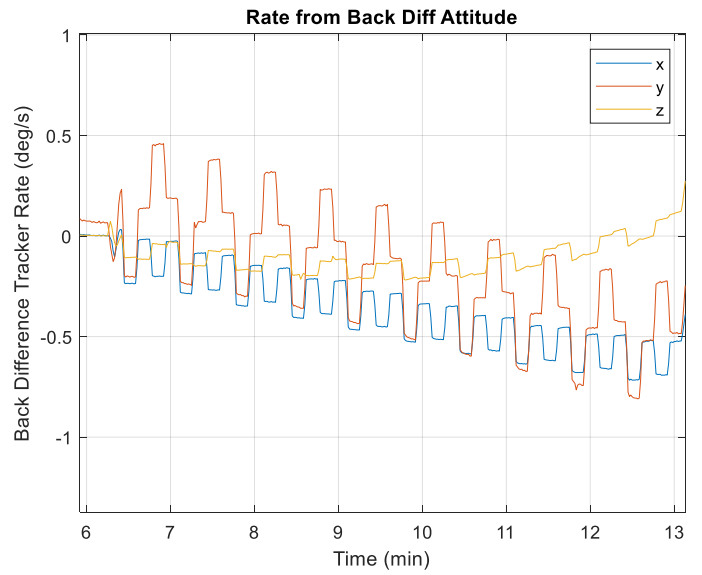

Figure 16: Rate Profile During Abbreviated Ibit Test

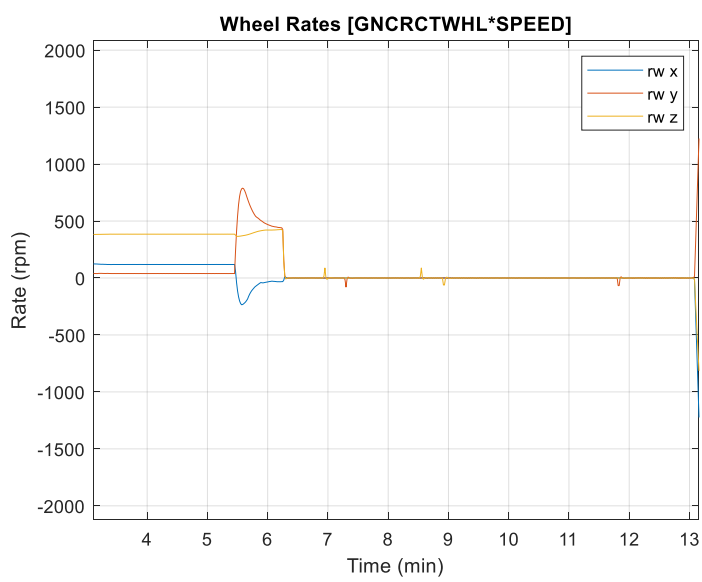

Figure 17: Reaction Wheels Commanded to 0 RPM Prior to Start of Ibit Measurement

With the abbreviated Ibit test completed, analysis of the thruster torques applied during the test showed that adjustment of the pulse widths would be beneficial for completing the full, 120 pulse, Ibit. Simulating the full 120 pulse Ibit based on the thruster torques measured during the abbreviated Ibit, facilitated in selecting a set of thruster pulse widths that would result in overall spacecraft motion that kept the star tracker clear of the Earth (Figure 20). Using pulse widths of [180 120200 180] msec for REAs 1-4 (Figure 19) enabled successful completion of the full 120 pulse Ibit test.

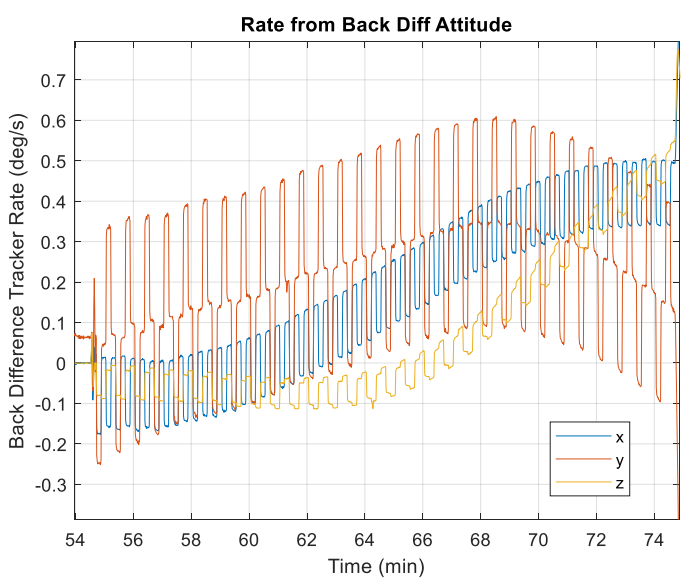

Figure 18: Rate Profile During Full Ibit Test Sequence 


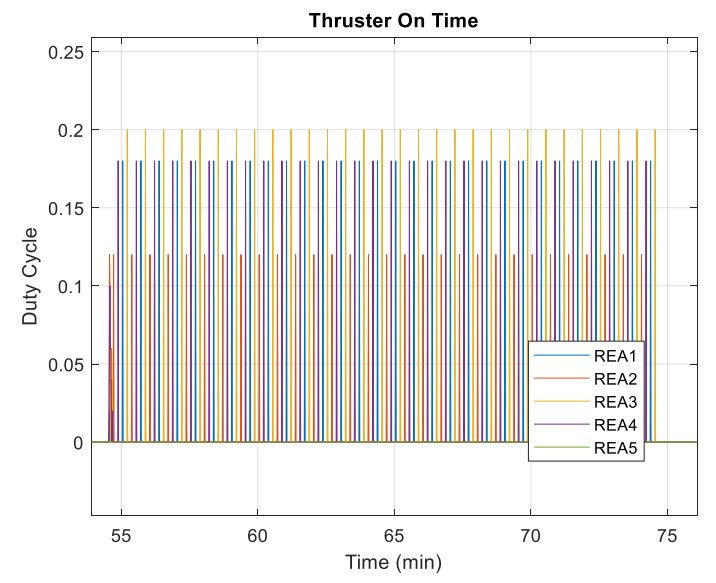

Figure 19: Commanded Thruster Duty Cycles During Ibit Test Sequence

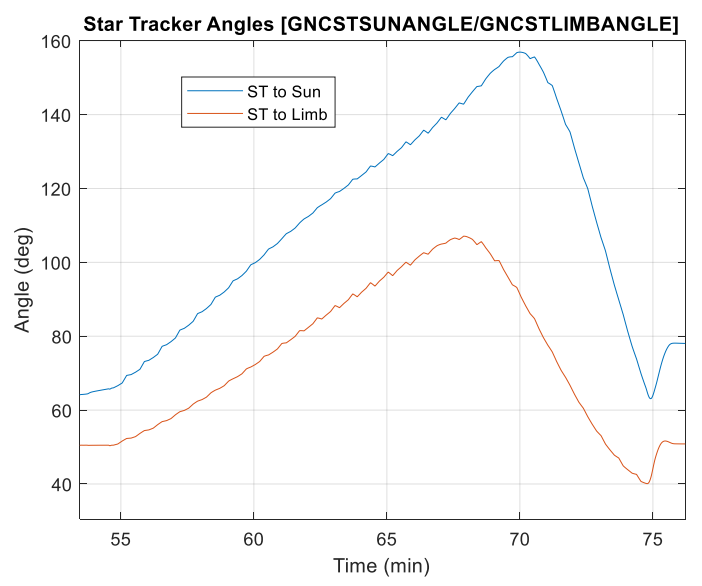

Figure 20: Star Tracker Motion Relative to Earth and Sun During Ibit Test

Prior to executing the longer duration delta-v's required to accomplish the first orbit lowering campaign, a 4 minute long delta-v checkout was performed to further evaluate pointing performance in a scenario where the thrusters have reached a steady state thrust level. This duration also allowed for any attitude errors incurred during thruster warm up to get corrected. With good pointing performance (Figure 21) and the desired thruster duty cycles maintained for the duration of the burn (Figure 22), the system was ready for the first set of operational orbit lowering burns.

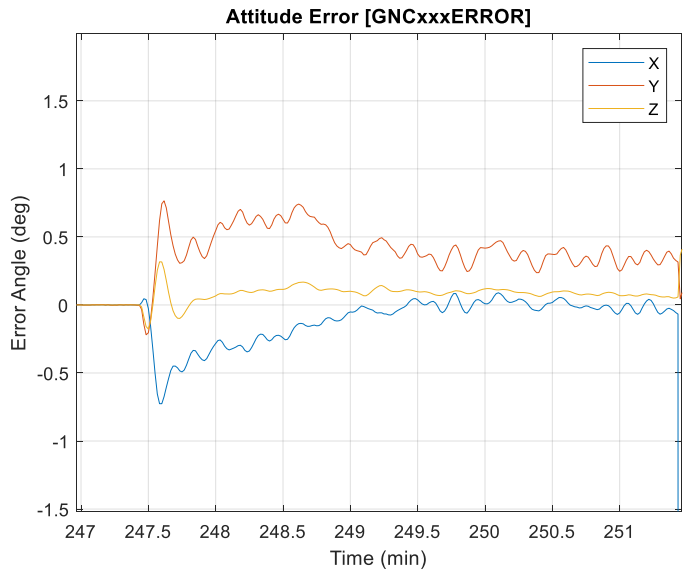

Figure 21: Attitude Pointing Performance During Delta-V Checkout

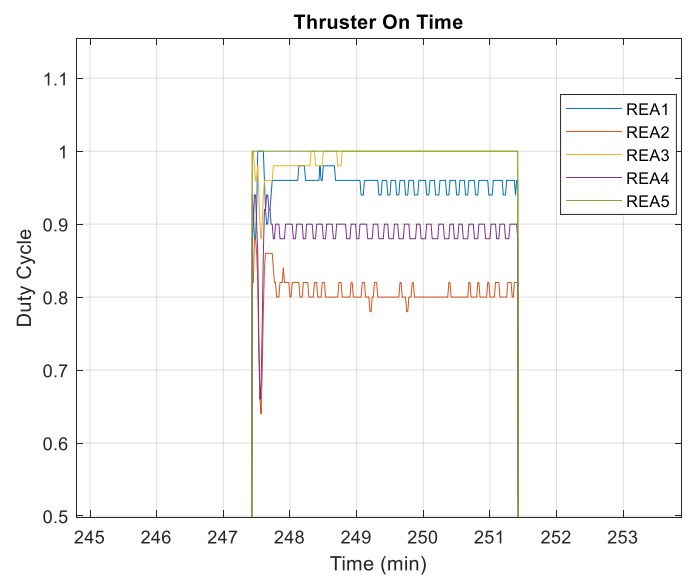

Figure 22: Thruster Duty Cycles During Delta-V Checkout

The first delta-v campaign was comprised of 3 individual burns of 6 minutes each, with the goal of lowering the perigee altitude to at least $575 \mathrm{~km}$. This altitude was targeted so that if no additional thruster burns ever get performed, GPIM would meet the 25 year re-entry timeline required of NASA missions in low Earth orbit. All of these burns were timed to start 3 minutes before an apogee crossing in order to maximize the efficiency of the delta- $\mathrm{v}$ application towards lowering perigee. Each burn performed as expected and the perigee altitude was eventually lowered to $545 \mathrm{~km}$ (Figure 23). 


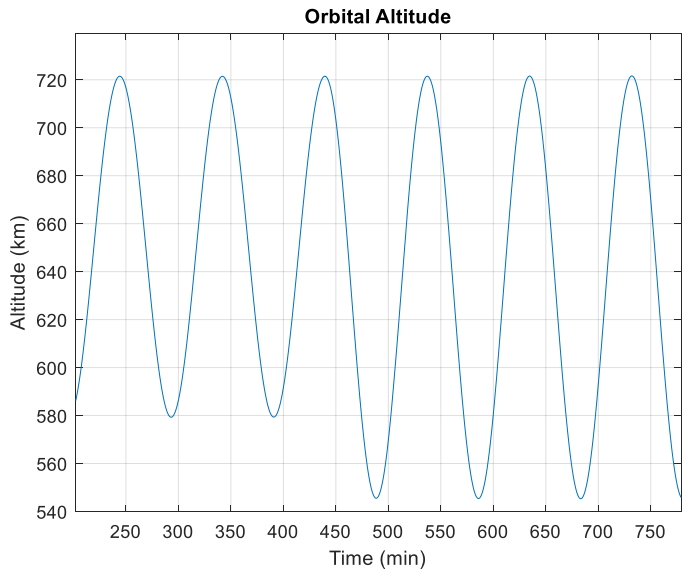

Figure 23: Orbital Altitude Change After Third Delta-V Burn

With the first orbit lowering completed, the next mission objective accomplished was performing the series of attitude control demonstrations. The first of these was simply holding attitude for an orbit on the ACS thrusters, with the reaction wheels and torque rods disabled. This test showed attitude pointing accuracy of better than 0.5 degrees (Figure 24).

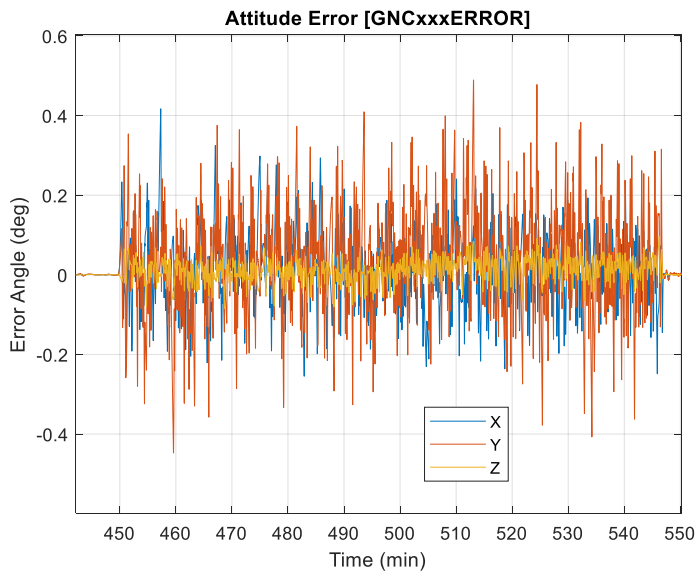

\section{Figure 24: Attitude Error during ACS Thruster Control Demo}

The second attitude control demonstration performed was managing reaction wheel momentum with the ACS thrusters. Naturally the torque rods were disabled for this demo, with the thrusters enabled to dump reaction wheel momentum. A series of maneuvers was performed on the reaction wheels with the torque rods disabled to put some momentum into the wheels. The thrusters were then commanded into the momentum management configuration, and responded by firing as needed to lower the wheel momentum (Figure 25 and Figure 26).

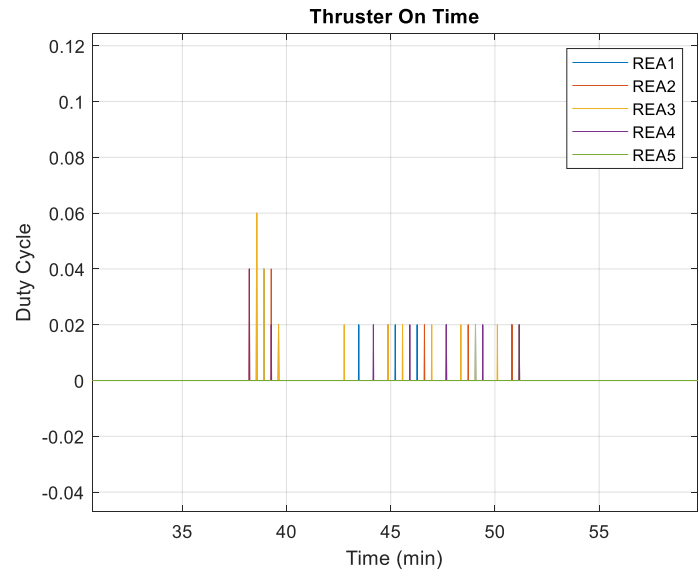

Figure 25: Thruster Duty Cycles to Manage Wheel Momentum

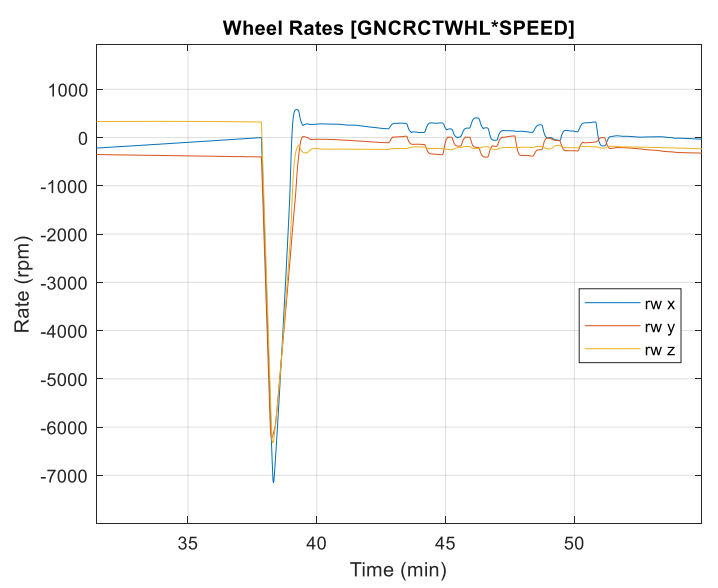

Figure 26: Reaction Wheel Momentum Managed by Thrusters

The next attitude control demonstration tested another new ADCS mode, called Thruster Detumble. This demo consisted of loading momentum into the overall system, meaning the spacecraft is moving at a non-zero rate and the reaction wheels also have some momentum. Since an accurate rate measurement is required for this mode to work properly, and the star tracker is the primary attitude sensor for GPIM, the demo was constrained to a single axis such that the star tracker would not be blinded during the demo. However, even with this limitation, the demo still showed that this propulsion system is capable of recovering a spacecraft that may have experienced a failure of some type that left it with saturated reaction wheels and in a tumble (Figure 27). 


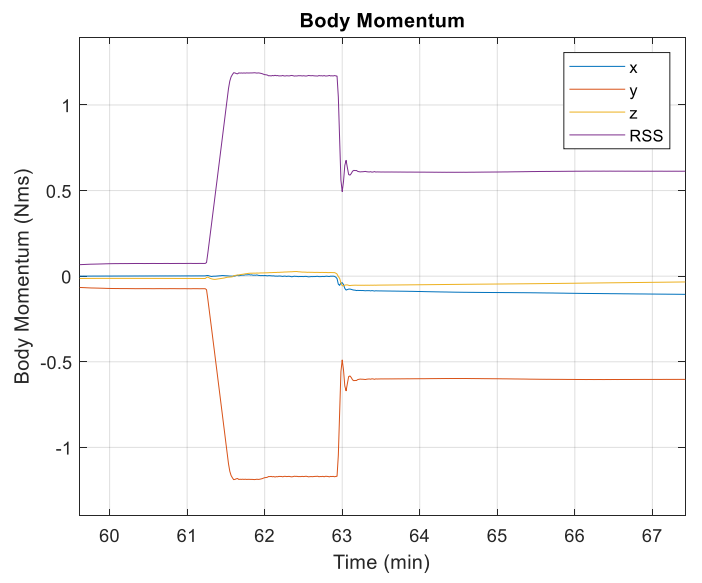

Figure 27: Overall Spacecraft Momentum During Thruster Detumble Demo

Finally, the last attitude control demonstration was done by performing a 60 second long delta- $v$ with the center thruster, REA 5, commanded to a $100 \%$ duty cycle and the corner thrusters set to off-pulse to provide thrust vector control (Figure 28). Because of the flexibility offered by the table parameter driven approach to flight software, reconfiguration of Delta- $\mathrm{V}$ Mode from onpulsing the ACS thrusters to off-pulsing them was as simple as commanding a table update.

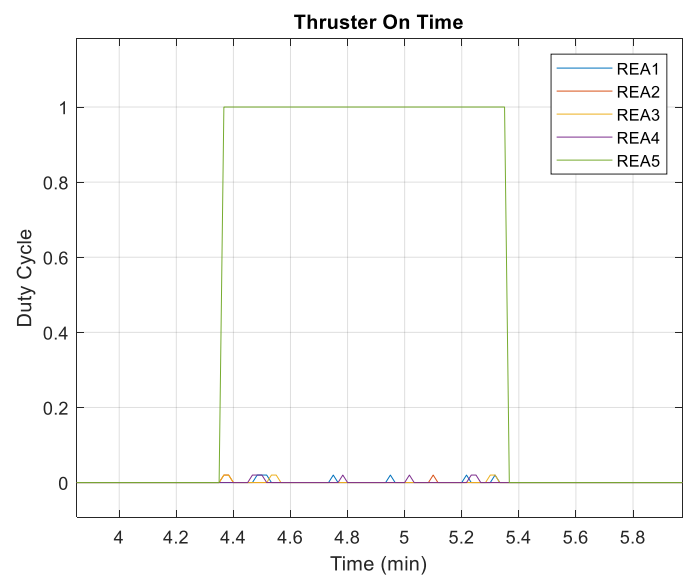

Figure 28: Off-Pulsing Delta-V Thruster Commands

\section{ONGOING FLIGHT ACTIVITIES}

As of this writing, a total of seven Ibit measurements have been performed, along with the necessary delta-v's to reach a $400 \mathrm{~km}$ perigee altitude. The SERB payloads have completed their checkouts and are in an operational state. Currently, a delta-v campaign is on-going to target a $300 \mathrm{~km}$ perigee altitude, with two more Ibit measurements to follow once this campaign is completed. The mission will be completed in late Summer 2020, after 13 months on-orbit, with the final orbit lowering burns targeting $180 \mathrm{~km}$, where it is expected that the spacecraft will re-enter within two weeks.

\section{CONCLUSIONS}

The Ball built GPIM spacecraft has provided a reliable platform for flight testing a brand new propulsion technology that offers increased safety and higher performance as compared to a traditional hydrazine system. On-orbit tests and demonstrations have followed a measured and systematic approach to proving the full flight system is capable of performing the complete suite of mission objectives. These mission objectives were developed to showcase a wide range of thruster-based operational capability and offer a measurement of thruster impulse bit as the system ages on-orbit. With the implementation of the optimized flight plan, the SERB payloads have the opportunity to collect valuable measurements of a wide range of upper atmospheric and space environment conditions on an orbit by orbit basis. Following completion of this 13 month mission, sufficient data will be have been accumulated to allow for infusion of AF-M315E as a hydrazine replacement for spacecraft attitude control and primary propulsion.

\section{ACKNOWLEDGEMENTS}

The Ball Aerospace GPIM TDM project team extends its thanks to NASA Marshall Space Flight Center for their support of this work under contract number NNM12AA79C. Ball Aerospace also thanks the propulsion group at NASA Goddard Space Flight Center for their peer review of the GPIM flight operations, and for their suggestions that have created a more robust flight program.

\section{REFERENCES}

1. C. McLean, et al, "BCP-100 Small Satellite Guidance Navigation and Control on the Green Propellant Infusion Mission", 2017 AAS GNC Conference, Breckenridge, CO, February 2017.

2. B. Marotta et al., "Green Propellant Infusion Mission Program Overview, Flight Operations, and ADCS Development," 2015 AAS GNC Conference, Breckenridge, CO, February 2015.

3. W. Deininger et al., "Implementation of the Green Propellant Infusion Mission (GPIM) on a Ball Aerospace BCP-100 Spacecraft Bus," 49th AIAA/ASME/SAE/ASEE Joint Propulsion Conference, San Jose, CA, July 2013.

4. Deininger, W. D., et al., "Description of the Green Propellant Infusion Mission," IEEE 2014 Aerospace Conference, Big Sky, MT, USA, 1-8 March 2014. 